\title{
Conformational changes in the peptidoglycan synthase activator LpoA are likely important for maintaining a viable cell wall in Gram-negative bacteria
}

\author{
Mark A Saper, Karthik Sathiyamoorthy, J Vijayalakshmi, Bhramara Tirupati, and Lixin Fan*
}

Department of Biological Chemistry and Biophysics Program, University of Michigan, Ann Arbor MI 48109-5606. *Frederick National Laboratory for Cancer Research, Leidos Biomedical Research Inc., Frederick, Maryland 21702

In many Gram-negative bacteria, the peptidoglycan synthase PBP1A requires the outer membrane lipoprotein LpoA to bind and activate the PBP1A transpeptidase enzyme for constructing a functional cell wall. Since RodA has recently been reported to be a major producer of peptidoglycan, the exact function of PBP1A is not known. Some hypothesize that one of its roles may be repair of the inevitable holes or gaps that appear during the cell elongation phase of growth. We determined the structure of three different crystal forms of LpoA from Haemophilus influenzae (HiLpoA) containing four independent full-length molecules. Unlike an elongated model previously suggested for $E$. coli LpoA, each $H i$ LpoA formed a U-shaped structure with a different relative $\mathrm{C}$-domain orientation. This resulted from both $\mathrm{N}$-domain twisting and rotation of the $\mathrm{C}$ domain (up to $30^{\circ}$ ) at the end of the relatively immobile interdomain linker. Similar $\mathrm{C}$ domain motions were also predicted by normal mode calculations. To ascertain the validity of these motions in solution, we collected small angle X-ray scattering data which confirmed a solution structure sampling structures equivalent to the X-ray and the normal mode calculated structures (see figure). A model will be presented where the flexibility of $H i \mathrm{LpoA}$ is essential for detecting large holes in the peptidoglycan and activating PBP1A to repair the holes.

\section{Relevant reference}

Sathiyamoorthy, K., Vijayalakshmi, J., Tirupati, B., Fan, L., and Saper, M.A. (2017). Structural analyses of the Haemophilus influenzae peptidoglycan synthase activator LpoA suggest multiple conformations in solution. J Biol Chem 292, 17626-17642.

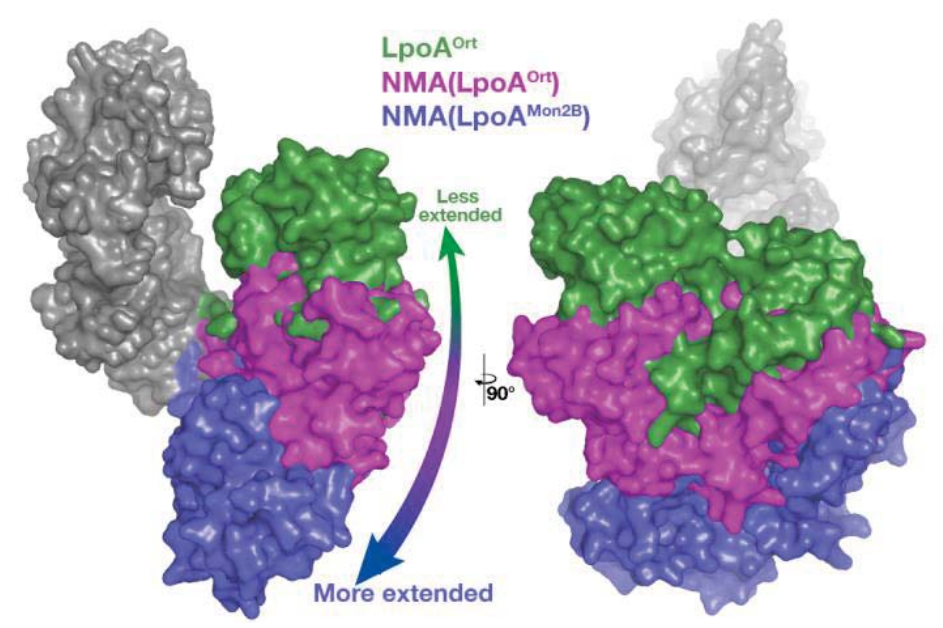

